\title{
CUltura e Viaggio. Vergine GiURATa di Elvira DoneS
}

\author{
Karol Karp \\ Università Niccolò Copernico di Toruń
}

\begin{abstract}
En): The aim of the article entitled Culture and journey. Vergine giurata by Elvira Dones is to present the role of two key motives, mentioned in the title, which appear in the work of Elvira Dones. They have a great influence on the life of the main character Hana. The culture demonstrates models which should be imitated and limits her individuality. She has to hide her female identity and to become a man, at least in the eyes of society. Leaving Albany turns out to be the only way of liberation. Hana goes to the United States to get to know herself again. The journey has two significations: the first concerns its fiscal dimension; the second is metaphoric and takes place in the internal world of the character. The analysis is carried out in relation to the concept of body which embraces such elements as sexuality and gender.
\end{abstract}

Key words: Elvira Dones; culture; journey; body; gender, identity

Parole chiave: Elvira Dones; cultura; viaggio; corpo; gender; identità

\section{Introduzione}

Daniele Comberiati individua due generazioni di autori migranti di origine albanese che adottano l'italiano come lingua d'espressione letteraria. La prima, rappresentata da Gëzim Hajdari (1957), ingloba scrittori che "hanno passato gran parte della loro esistenza sotto il regime di Hoxha e che solo negli anni Novanta hanno avuto la possibilità di scrivere senza essere censurati”, la seconda invece quelli che hanno iniziato a comporre le loro opere dopo il crollo della dittatura (COMBERIATI, 2013: 29). Di quest'ultimo gruppo fa parte tra l'altro Elvira Dones (1960), il cui romanzo Vergine Giurata si intende indagare in questa sede. Il perno della trama dell'opera sembra essere costituito da due motivi: quello della cultura e quello del viaggio, per cui mireremo a precisare dettagliatamente la loro funzione.

\section{Nella prigione della cultura, ossia nella prigione del corpo}

Risulta d'obbligo mettere subito in risalto che la cultura presentata nell'opera si dimostra molto diversa da quella comunemente intesa come occidentale. Per tale motivo, analizzandola, è opportuno ricorrere al metodo di ricerca proposto dall'imagologia. Essa, come afferma Nora Moll, "può essere intesa come una delle forme di indagine più "concrete" dell'approccio con l'alterità." (MOLL, 2002 : 185) poiché consiste nell' "immaginare altri paesi, formulare giudizi sui loro abitanti, confrontare sé e i propri connazionali con gli altri, gli stranieri." (MOLL, 2002 : 185). Per imagologia "si intende lo studio delle immagini, [...] in generale delle opinioni su altri popoli e culture che la letteratura trasmette." (MOLL, 2002 : 186).

Con l'opera in esame ci si inoltra nella visione del codice culturale, del cosiddetto Kanun, che regola la vita della comunità albanese ivi descritta. Si tratta di un insieme di leggi tramandate di generazione in generazione, quindi fortemente radicate nella tradizione. La presenza di un codice, come rileva il filosofo polacco 
Leszek Kołakowski, caratterizza ogni mondo conservativo, (KOŁAKOWSKI, 1967 : 163) e quello delineato da Dones è sicuramente tale.

Margaret MEAD, nel suo libro Culture and commitment. A study of a generation gap (1970), prendendo in considerazione chi stabilisce i modelli che vanno seguiti all'interno di un gruppo sociale, annovera tre tipi di culture: postfigurativa, configurativa e prefigurativa. Seguendo tale distinzione va constatato che la cultura presentata nell'opera analizzata è post-figurativa, in quanto si impernia su modelli provenienti dal passato, comunemente considerati giusti. (MEAD, 1970 : 25) Essi, come detto, sono raccolti nel Kanun, che assegna ad ogni sesso, e ciò ci interessa particolarmente, ruoli e comportamenti ben precisi. Così il sesso biologico si trasforma in sesso culturale. Ciò avviene perché esso risulta totalmente dipendente dalla cultura (BUTLER, 2008: 52-58). La storia della protagonista costituisce un buon esempio di quanto appena rilevato e attesta la correttezza della teoria gender propagata dalla studiosa americana Judith Butler. Il sesso diventa sinonimo di imprigionamento (BUTLER, 2008: 216). La teoria gender si dimostra come il modo più fondamentale e universalmente accettato di dividere e catalogare gli esseri umani (MEADOws, 2012 : 290). Hana, orfana in giovane età, viene cresciuta dai parenti. Lo zio Gjergj, essendo gravemente malato e cosciente del suo stato, comincia a cercare un uomo che si prenda cura di lei, poiché, secondo la tradizione, una donna solitaria non va rispettata.

\footnotetext{
"Resterai sola”, dice lentamente Gjergj. "Una donna non sposata non vale niente." "Una donna è uguale a un uomo."

"Balle! La donna è fatta per servire l'uomo e dargli dei figli. Non osare!" [...]

"Pensavo tu fossi diverso," dice tra i denti. Non è sicura che lui abbia sentito, perché non reagisce.

"Resterai sola," ribadisce, "e non voglio lasciarti indifesa. [...] Sei solo una donna."
}

(DONES, 2009 : 102-103)

Gjergj rileva l'importanza dell'uomo nella società albanese. La sua presenza dà alla donna uno status e le consente di funzionare all'interno della comunità a cui appartiene. Solo lui è in grado di occuparsi bene della kulla. Va aggiunto che in tale modo viene chiamata "una tipica abitazione albanese a forma di torre" (PELLEGRINI, 2013 : 152). Lo schema sociale tratteggiato nel romanzo è dunque imperniato sulla figura maschile. Ciò non significa però che la donna sia disprezzata oppure maltrattata. Al contrario, accompagnata da un uomo può essere sicura della sua posizione. Lui svolge il ruolo del suo protettore e del suo sovrano contemporaneamente. Il controllo che gli viene dato, come rileva Cynthia Fuchs Epstein, attesta la sua superiorità (FUCHS EPSTEIN, 2012: 30). Vale la pena mettere in evidenza che nell'opera di Ornela VORPSI (1968), un'altra scrittrice migrante di origine albanese, dal titolo Il paese dove non si muore mai (2005), abbiamo anche a che fare con una società fortemente maschilista. A differenza di Dones però, l'autrice si concentra sul carattere distruttivo del dominio degli uomini, il quale non fa delle donne che oggetti da guardare e consumare. In Vorpsi la donna è percepita come un mezzo per soddisfare i bisogni sessuali dell'uomo, nel romanzo in esame invece lei risulta un essere debole, la cui esistenza non ha 
senso senza la sua protezione. Nel primo caso ci si avvicina più a un contesto erotico, nel secondo a quello sociale.

Hana, sebbene inizialmente si opponga alla tradizione, in fin dei conti decide di accettare "la posizione di debolezza, tutta femminile" (PELLEGRINI, 2013 : 153), si traveste da uomo e diventa "vergine giurata". Attraverso la sua decisione dimostra però molto coraggio. Da un lato si sottomette al codice sociale, dall'altro quello che fa esige un grande sacrificio. E poi si scopre che ciò influirà su tutta la sua vita futura.

Quando scende le scale e si presenta a Gjergj vestita da uomo, lui resta ammutolito. Il mento gli si muove, di colpo, la mascella serrata non lo aiuta a fermare la commozione. È il 6 novembre 1986. Hana graffia la data sulla parete della stanza degli ospiti. [...] Quando ha finito, torna ancora da zio Gjergj. Lui le allunga il fucile. Lei lo prende in mano. Lo esamina. Appartiene a sei generazioni di uomini del clan Doda. Gjergj l'ha oliato per trentasei anni. Hana resta in piedi, goffa. E adesso, si domanda, e adesso? Adesso niente. [...] Dovrebbe sedersi da uomo, a gambe incrociate, dovrebbe fumare una pipa con Gjergj. [...]

"Sei sicura di questo passo, figlia?"

“Mi chiamerò Mark. Sarò Mark Doda.” (DonEs, 2009 : 111)

Franca Pellegrini, spiegando la scelta fatta dalla protagonista, mette in evidenza che secondo la tradizione albanese "una ragazza che rimane orfana" (PELLEGRINI, 2013 : 151) ha due alternative: "accettare un matrimonio combinato o diventare 'uomo', assumendone le sembianze sociali." (PELLEGRINI, 2013 : 151). Hana, desiderando scappare dalla necessità di vivere con un uomo non amato, cosciente delle conseguenze, sceglie la seconda possibilità. Gjergj, trasmettendole il fucile, il simbolo del potere maschile, esprime l'accettazione della sua decisione, consentendole di chiudersi nella prigione della propria carnalità. Va sottolineato che il corpo sarà l'unica prospettiva attraverso cui verrà percepita dai membri della società. Esso diventa, come afferma Elisabeth Grosz, un testo che può essere decifrato da altri e che svela lo stato psichico dell'individuo, essendo al contempo portatore di codici sociali (GROSZ, 1995 : 2934). Il corpo, costituente il segno visibile della stigmatizzazione dell'uomo, riflette la legge imposta dalla società. (GALIMBERTI, 2003 : 370-372). Hana diventa oggetto di analisi. Il suo esterno suscita riflessioni, ma non provoca stupore, in quanto tutti, conoscendo le leggi tradizionali, sono in grado di comprendere l'atteggiamento della ragazza. Attraverso la sua immagine esteriore lei svela una parte considerevole della sua interiorità. La protagonista di sicuro si sente smarrita nel ruolo che è tenuta a interpretare. Seguendo Sigmund Freud, che rileva il ruolo oppressivo della cultura, va constatato che è proprio essa a costituire la fonte delle sofferenze di Hana (FREUD, 1992 : 10). Lo stato della protagonista è ben visibile nel suo dialogo con Blerta, un'amica dell'università che inaspettatamente arriva a casa sua.

\footnotetext{
"Raccontami di questi anni, Hana, vuoi?" la invita. "Raccontami com’era. Com’è."

"Non c'è niente da raccontare."

"Dieci anni della tua vita e non c'è niente da raccontare?"

"No." [...]

Hana ride, con le lacrime che riaffiorano e il cuore che cerca invano di restare indifferente.
} 
"Non si può raccontare la propria morte. Trovami un cadavere che ci sia riuscito e io mi levo il cappello." (DONES, 2009 : 157)

Freud mette in risalto quanto la cultura influisca sull'individuo facendolo soffrire, ma paradossalmente tutto ciò che considera come un mezzo di difesa per attenuare il dolore appartiene alla cultura stessa (FREUD, $1992: 76$ ). Nel caso della protagonista di Dones abbiamo a che fare con un tipo di sospensione: la sua vera vita, élan vital come direbbe Henri Bergson, deve fermarsi di fronte alla nuova identità culturale racchiusa nel corpo maschile (KUPER, 2005: 202). Esso si presenta con una doppia funzione: da un lato ingabbia la sua natura, la sua femminilità, dall'altro visualizza l'accettazione del codice sociale che la costringe a immergersi nel mondo degli uomini. Va aggiunto che farsi uomo significa per Hana cominciare a compiere azioni completamente diverse da quelle abituali, può ad esempio bere il raki e fumare la pipa.

Nel quadro della vita della protagonista delineato nel romanzo spicca la presenza del determinismo, la quale è generalmente molto visibile nelle opere degli scrittori contemporanei in lingua italiana dalle radici albanesi. Nelle opere di Carmine Abate (1954), come ad esempio Il ballo tondo (1991) e La festa del ritorno (2004), incontriamo numerosi protagonisti di provenienza arbëresh, che, vista la specificità di essa, sono condannati a seguire comportamenti ben precisi. Sono sovente costretti ad abbandonare la propria comunità in cerca di mezzi per vivere, diventano esuli e fronteggiano le difficoltà dell'emigrazione (Bovo RomoeUf, 2008 : 71). Nel romanzo Fuorimondo (2012) di Vorpsi il determinismo riguarda i ruoli che sono assegnati agli individui, le capacità e i tratti dati loro nel momento di nascita:

Certa gente era giorno e notte madre come Maria, certe donne erano nate per essere mogli come Hera e lavare tutti i panni di una famiglia senza fine. La gatta avrebbe portato a casa i cuccioli ogni autunno, Dolfi sarebbe stato sempre giovane, i suoi denti eternamente bianchi. Le ragazze avrebbero continuato a bussare alla porta, io Tamar sarei sempre stata una spettatrice e ogni tanto avrei sentito quel brivido strano che mi percorre il cranio e mi allontana da tutto. Nasciamo e tutto è già determinato, i ruoli sono distribuiti. (VORPSI, $2012: 20$ )

Per Anilda Ibrahimi (1972), e pensiamo all'opera intitolata Rosso come una sposa (2008), la sorte dell'uomo è condizionata dalla cultura in cui cresce, fortemente influenzata dal retaggio degli avi, ossia dalla tradizione. Il medesimo stato di cose caratterizza il romanzo in esame. La cultura, come detto, tratteggia il cammino esistenziale di Hana, ma non solo. Essa determina completamente anche le azioni di Gjergj, che, occorre dirlo, muore fedele alla legge della sua terra. In quanto uomo, a cui è riservata una posizione più favorevole rispetto a quella della donna, non sente il bisogno di liberarsene. Hana invece, dopo tanti anni desidera ricominciare a vivere appieno. A questo punto la sua situazione fa pensare alla teoria di Leszek Kołakowski. Il filosofo considera il mondo come l'eredità data all'individuo dagli antenati, di cui è possibile, dopo qualche tempo, mettere in questione il valore. Rifiutare il mondo significa rifiutare la vita. Accettarlo però significa accettare tutti gli elementi negativi che vi sono inerenti (KOŁAKOWSKI, 
1967 : 149). La protagonista non rifiuta la vita stessa, in quanto non sceglie il suicidio, ma non può più vivere in sintonia con le esigenze della realtà circostante che la opprime. Abbandona il suo microcosmo, il suo retaggio culturale ed esistenziale per recarsi negli Stati Uniti, dunque in una dimensione completamente nuova, sperando di riscoprire la sua femminilità.

\section{Il viaggio verso la libertà}

Carla Fratta, facendo ricorso al senso che il termine identità riveste "nell' ambito delle culture letterarie postcoloniali" (FRATTA, $2001: 45)$, accenna al fatto che l'identità è fra l'altro "un dato convenzionale, espressione simbolica di una cultura, luogo virtuale e progettuale definito a partire da un insieme di

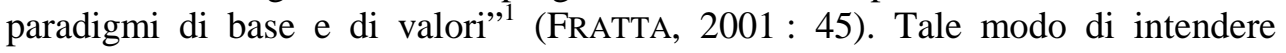
l'identità va perfettamente d'accordo con la situazione della protagonista di Dones. Con la decisione di farsi uomo Hana adotta la nuova identità, quella che le viene imposta dalla cultura. Riferendoci a Paul Ricoeur, possiamo chiamarla ipse (RICEUR, 1990 : 167-168). La sua identità idem però, quella femminile, non si spegne mai e paradossalmente viene riportata in vita in America. Il caso della protagonista risulta particolare poiché di solito giungere in un paese straniero fa apparire e svilupparsi l'identità ipse, quella mutabile, influenzata dal processo di ambientazione dell'individuo in seno a una società diversa. L'identità ipse di Hana però nasce nella sua propria terra, in cui è costretta a vivere estranea a se stessa. Il viaggio negli Stati Uniti, intrapreso dopo tanti anni di imprigionamento, la porta a ritrovare la sua vera identità, quella idem. Il ritrovamento di essa non deriva dall'incontro con l'alterità (CHANCÉ, 2005 : 18), è possibile solamente fuori dall'Albania, in uno stato dove il Kanun perde la sua forza, è il risultato dei bisogni interiori, del fatto che nella sua interiorità rimane sempre vivo il suo spirito femminile.

La riscoperta identitaria della protagonista avviene a tappe. Si tratta di un lungo processo in cui sono coinvolte più persone, in quanto, come sostiene Adam Kuper "l'identità non è solamente un problema personale. Deve essere vissuta nel mondo del dialogo con gli altri. [...] Da un punto di vista soggettivo l'identità si svela nell'interiorità di un individuo, e da ciò risulta l'identità con gli altri’’2 (KUPER, 2005 : 203). La protagonista giunge negli Stati Uniti in quanto uomo, il suo aspetto esteriore tradisce però dei tratti femminili. Lila, la cugina di Hana, che la ospita è cosciente che ciò può provocarle dei problemi poiché in America "non ti assumono, se hai un aspetto insolito. Nessuno vuole problemi, solo braccia da lavoro più normali possibili." (DONES, 2009 : 47). Dalla citazione si evince che in America i giudizi sugli altri si esprimono nell'ottica del corpo, che, come in Albania, costituisce il primo punto di riferimento nei contatti interpersonali. Di nuovo Hana si trova davanti al dilemma della propria carnalità, ma stavolta, per essere accettata dalla società, deve farsi donna e ciò esteriormente è più facile.

\footnotetext{
${ }^{1}$ Oltre alla definizione suddetta la studiosa sottolinea che nel contesto in oggetto l'identità è strettamente legata al concetto di alterità. In più può essere intesa come dato soggettivo (sentimento di identità) e oggettivo (capacità di riconoscimento dell'identità altrui) (FRATTA, 2001 : 46).

${ }^{2}$ La traduzione è a cura dell' autore del saggio.
} 
Prima di rimettersi nei panni di donna parla alla figlia di Lila, Jonida, che da sempre la considera come lo zio Mark.

La ragazzina si apre in un sorriso ed estrae una sigaretta. Poi si infila il pacchetto in tasca.

"Chi sei veramente, allora, zio Mark?"

"Sono tua zia, prima cugina di tua madre Lila."[...]

"Io lo so che sei gay," dice poi con tono intimo, "questo si capisce. Però..."

"Aspetta", la ferma Hana. "Io, gay?"

"Che cosa, se no? Perché tutto questo mistero su di te? Sei omosessuale, l'avevo pensato subito io." [...]

"Non sono un uomo. E non sono gay, nemmeno un po'. Sono proprio donna. Sono donna dalla nascita. [...] Non sono gay, e nemmeno lesbica," ripete. So di sembrare strana, una specie di ibrido, ma sono una donna."

"E il seno dove c'è l'hai?"

"C'è, piccolo ma c'è. Porto camicie larghe, come vedi." [...]

"Be', adesso non capisco proprio niente. Cominciamo dal perché ti vesti e ti comporti da uomo. E come hai fatto a sembrare un uomo, anche se strano." (DONES, $2009: 51-52$ )

Nei testi dell'Ottocento, come osserva Michel Foucault, l'omosessualità maschile è segnata da tratti posseduti dall'uomo che rinviano al femminile: costruzione del corpo, gesti, vestiti (FOUCAULT, 2000: 157). Va rilevato che proprio essi fanno pensare alla figlia di Lila che Mark sia gay. L'atteggiamento di Jonida è anche in sintonia con le affermazioni della teoria queer (ATKINS : 1998), secondo cui: "quello che indossiamo e come usiamo i nostri corpi" ${ }^{3}$ (MEADOWs, 2012 : 295) può svelare il nostro orientamento sessuale. Così al corpo viene assegnata una categoria precisa, un'etichetta che può essere una forma di organizzazione del potere (FOUCAULT, $2000: 46$ ). La possibilità di catalogare il corpo, intenderlo stigmatizzato oppure perverso, allarga il controllo, intensifica la limitazione (FOUCAULT, 2000 : 46). Esso, ancora una volta, si presenta come un aspetto del soggetto che blocca le linfe vitali e lo espone a giudizi ingiusti.

Vale la pena di mettere in evidenza che la tematica omosessuale appare nella letteratura italiana contemporanea sempre più di frequente. Basta evocare il gruppo dei cosiddetti "cannibali" oppure, come li chiama Barbara Kornacka, "giovani scrittori" degli anni Ottanta e Novanta, i quali trattano il motivo dell'omosessualità in modo molto audace (KORNACKA, 2013: 211). Fra loro spiccano due autori, Pier Vittorio Tondelli (1955-1991) e Aldo Busi (1948) che "come primi scrittori italiani parlano in modo aperto del loro orientamento sessuale, [...] introducendo numerosi e complessi motivi omosessuali e transessuali ${ }^{4} . "$ (KORNACKA, $2013: 238$ ). Sembra opportuno mettere in evidenza che Hanna Serkowska, citata da Kornacka nel libro Orecchio, occhio, corpo. Sulla narrativa dei "giovani scrittori" degli anni Ottanta e Novanta in Italia (2013), analizza il filone omosessuale nella lettaratura italiana del secondo Novecento accennando alla sua evoluzione (SERKOWSKA, 2005).

\footnotetext{
${ }^{3}$ La traduzione è a cura dell' autore del saggio.

${ }^{4} \mathrm{La}$ traduzione è a cura dell' autore del saggio.

${ }^{5}$ La traduzione a cura dell' autrice del libro (KoRNACKA, $2013: 271$ ). Il titolo originale in polacco è Ucho, oko, ciato. O prozie "młodych pisarzy" lat osiemdziesiatych i dziewięćdziesiątych we Wtoszech.
} 
Il giudizio sulla sessualità dello zio espresso da Jonida si costruisce esclusivamente in riferimento alla carnalità di lui. Ancora una volta il corpo si dimostra come un'etichetta dell'individuo agli occhi altrui. Esso costituisce un testo che viene letto dagli altri (GROSZ, 1995: 29-34). La lettura effettuata dall'adolescente non la porta però a scoprire la verità che, una volta rivelata, la sciocca profondamente. Così ci troviamo di fronte a un corpo che inganna e provoca impressioni sbagliate.

Hana racconta alla ragazza la storia della sua vita. La figlia di Lila, che non conosce affatto la cultura degli antenati, stenta a comprenderla. A questo proposito Franca Pellegrini, attestando al contempo la presenza dell'autobiografismo ${ }^{6}$ nell'opera in esame, giustamente osserva che Dones intende:

(...) sottolineare la difficoltà di incontro fra mondi ancestralmente separati ed è come se si creassero sulla pagina, intercalati fra loro, due canali comunicativi: uno fa capo all'origine della scrittrice e l'altro che risponde pragmaticamente al canone occidentale, o all'interpretazione di esso. Il tentativo di Hana di raccontare a Jonida in parallelo la sua storia e la storia del suo paese, corrisponde al tentativo della Dones di mettere in comunicazione la sua terra di origine con la terra di adozione. (PellegRini, 2013 : 155)

Dopo la conversazione con Jonida, un passo significativo nel processo di riscoperta della sua identità, Hana si sente libera di incominciare a vivere di nuovo da donna. Ciò significa soprattutto diventare sicura nei confronti della propria sessualità, del proprio corpo. Secondo Zygmunt Bauman l'obiettivo del corpo postmoderno è quello di sperimentare varie sensazioni. Esso si apre pienamente a impulsi che provengono dal mondo esterno, li assorbe e li digerisce (BAUMAN, 1995 : 90-98). Così diviene un mezzo attraverso cui l'individuo è capace di provare piacere, un elemento naturale della sua soggettività (ADORNO, 1999 : 66). Se il corpo cerca piacere, lo si può definire sano. Il corpo sano intende impossessarsi di piaceri variegati: sessuali, gastronomici, visivi oppure auditivi (BAUMAN, 1995 : 91). Il corpo malato invece vi rinuncia manifestando apatia, mancanza di desideri, indifferenza alle tentazioni (BAUMAN, 1995: 90). Conformemente alla teoria suddetta, possiamo constatare che il corpo di Hana si accosta allo stato di salute proprio in America. Nel periodo della sua vita che va dal giuramento alla partenza, si presenta malato poiché è privo di brama. Alla vergine giurata non viene permesso di avere rapporti sessuali. Se lei non rispetta la legge e soccombe alla tentazione rischia di essere ammazzata. Hana rifiuta il sesso poiché costretta dal codice sociale, e non dalle regole della moralità che di solito, come afferma Foucault, spingono a fare sacrifici (FOUCAULT, 2000 : 161). Non

\footnotetext{
${ }^{6}$ L'autobiografismo può indubbiamente essere considerato uno dei tratti distintivi della produzione degli autori migranti. Di solito esso si estende a molti aspetti che vanno dalla migrazione vissuta dagli stessi scrittori, attraverso alcuni motivi personali, al messaggio di carattere imagologico. Nel romanzo in esame si percepiscono tutti questi elementi. Dones lascia l'Albania nel 1988 per recarsi prima in Svizzera, poi negli Stati Uniti, dove vive finora. È possibile identificare la sua esperienza migratoria con quella di Hana. Inoltre la trama fornisce numerose informazioni sulla cultura albanese, ben conosciuta dall'autrice. A questo punto vale la pena accentuare che Maria Cristina Mauceri analizza la presenza dell'autobiografismo nel romanzo di Dones Senza bagagli (1997), uscito prima in albanese con il titolo: Dashuri e huaj, poi tradotto in italiano.
} 
possiamo affermare però che a Hana-Mark il tema del sesso non interessa affatto. Lo si vede nel dialogo con Blerta:
Si siedono, con i bicchieri pieni.
"Allora, alla tua salute, uomo di casa!" grida Blerta.
Hana infila il naso nel bicchiere e annusa il vino. Poi guarda l'amica.
"Com'è il sesso?" domanda a bruciapelo. [...] Tu hai vissuto con un uomo, com'è? [...]
Dimmi semplicemente com'è" insiste Hana. "Tra il niente di speciale, la delusione di una volta di tante donne albanesi, e la libertà sessuale oggi nelle città, che peso ha realmente il sesso nella vita quotidiana? Per esempio per una come te, Blerta. Com'è.” [...]
"È bello. La mia esperienza è stata bella," dice con naturalezza.
"Vale la pena provare, allora?"
"Vale la pena provare, Hana, sì."
"Bene," sospira lei sollevata. (DONES, 2009 : 159-160)

Per scoprire il mondo del sesso, ossia avere un rapporto con un uomo, e recuperare appieno la sua identità femminile, Hana si concede del tempo per prepararsi. E ciò, come detto, è inerente alla sua carnalità. La protagonista guarda attentamente il suo fisico nello specchio e pratica onanismo, dispone del corpo che si presenta come la sua "proprietà privata" (BAUMAN, 1995 : 94). Tale situazione però, secondo Bauman, risulta problematica. Il corpo si immerge nel mondo delle sensazioni per provare piacere, ma la mente dovrebbe controllarlo. (BAUMAN, 1995 : 94) Tale dipendenza tra corpo e interiorità indubbiamente caratterizza il personaggio di Hana. Attraverso l'atto dell'onanismo lei mira a un piacere mai conosciuto prima. Va anche detto che quest'azione deriva dal suo obiettivo principale, ossia dalla volontà interiore di conoscere se stessa, di riavere la sua femminilità. Il piacere sessuale è portatore di forza che assume una funzione conoscitiva (FOUCAULT, 2000: 445). Sperimentando con il corpo si arriva a comprendere meglio il proprio essere, la propria soggettività (FOUCAULT, 2000 : 445). Le azioni che l'individuo esercita sul suo corpo sono un elemento della cosiddetta "cultura di se stesso" (FouCAULT, 2000: 445). Essa lo porta a soddisfare i suoi bisogni di ogni tipo, ben compresi quelli sessuali. Nel caso di Hana l'onanismo, ossia la cura di se stessa, costituisce una tappa rilevante nel ritrovamento dell'identità, il cui punto culminante sarà il rapporto con un uomo. Da ciò si evince che il sesso svolge un ruolo molto positivo nella vita dell'individuo $^{7}$. (FOUCAULT, 2000 : 490) La sua fisicità, la sua carnalità, hanno un impatto considerevole sulla sua interiorità.

Le mani di Patrick passano sul suo seno con lentezza ma senza fermarsi. Scivolano oltre, vanno sui fianchi e ci restano. Hana ci mette del tempo prima di lasciarsi andare. Lui continua ad accarezzarla e a baciarla, mentre lei si sforza di capire se le piace. Ha il terrore di ricambiare le carezze di Patrick, perciò si aggrappa al lenzuolo e si sente più sicura. [...] Patrick torna su di lei e lascia che il suo corpo aderisca a quello di Hana. [...] Lei scivola fuori dal letto solo quando il respiro di Patrick si è fatto profondo. Si veste ed esce dall'appartamento. Guida verso casa tutta concentrata, fuma la sigaretta che da un

\footnotetext{
${ }^{7}$ Vale la pena di mettere in evidenza che Foucault si pronuncia anche, in prospettiva diacronica, sulla funzione negativa del sesso. Esso può provocare esaurimento fisico e addirittura alcune malattie (FoucAult, $2000: 491$ ).
} 
pezzo conserva per questo dopo. La notte è deserta e stranamente stanca. Lei no. Si sente sveglia, e appena arriverà a casa fumerà ancora. Adesso sa che ne avrà di vita da vivere, qualsiasi cosa succeda d'ora in poi. E prima che sia giorno, dormirà. Prima che sia ancora paura. Ma questa non la teme, tutt'al più la detesta solo un po'. Ha sentito il proprio corpo reagire, lo ha sentito pulsare.

"Benvenuto in me, cretino d'un corpo," dice a voce alta. Getta il mozzicone dal finestrino. È bello sapere di non dover morire. (DONES, 2009 : 203-204)

Il rapporto sessuale, un tipo di happy end, che la protagonista vive con Patrick, l'uomo che si guadagna la sua fiducia, e a cui affida la sua storia, segna l'ultima tappa nel processo di ricostruzione della sua identità femminile. In esso spicca il ruolo fondamentale del corpo che reagisce correttamente al segnale emesso da un rappresentante del sesso maschile, del corpo che accetta le sue carezze e vi risponde. Hana si sente di nuovo sicura di chi è, riesce a identificarsi con la propria carnalità, sembra considerarsi vincitrice nella battaglia in cui il suo corpo e la sua anima sono coinvolti da tanto tempo. Rappresentare fisicamente un sesso, essere visti da altri come rappresentanti di questo sesso, provare una precisa identità sessuale sono elementi che devono costituire una struttura omogenea affinché l'individuo possa vivere. Solo quando queste tre zone vanno d'accordo la protagonista spera bene per il futuro, o meglio, vede un futuro. Non importa come sia, importa che lei sia in grado di percepirsi come un essere unito.

\section{Conclusioni}

I due elementi che costituiscono il perno del romanzo analizzato, la cultura e il viaggio, sono inseparabilmente legati al concetto di corpo. Dones mira a visualizzare il carattere oppressivo della cultura albanese, codificata nel Kanun, attraverso il corpo che diventa la prigione delle passioni e della femminilità di una donna, a cui viene assegnato un ruolo completamente opposto alla sua natura, un ruolo lontano dalla normalità, ossia quello maschile. Nella storia presentata imbevuta di elementi controversi, attraverso le gravi conseguenze che risultano dalla trasformazione di Hana in Mark, si intuisce l'intenzione di denunciare l'ingiustiza di tale situazione. L'individuo, libero per natura, a causa delle implicazioni culturali, fronteggia la necessità di chiudersi in un carcere. Esse si dimostrano troppo forti per poterle rifiutare. Occorre aggiungere però che la loro validità si estende solamente sul territorio dell'Albania, in un altro stato non sono più in vigore. Andare in una terra più ospitale, ossia fare un viaggio, diventa l'unico mezzo per liberarsi. Per Hana in America comincia il processo opposto a quello che deve vivere nel suo paese natale: da uomo si trasforma in donna per svelare al mondo e a se stessa i meandri del labirinto della sua identità. Il periodo di tale trasformazione risulta di sicuro estremamente difficile, è intriso di dubbi, di insicurezze e di timore. Esso, metaforicamente, come osserva a ragione Maria Cristina Mauceri, può essere definito come un viaggio di ritorno al suo gender originario (MAUCERI, 2009 : 87). Nella trama del romanzo la studiosa annovera anche un altro viaggio, quello fisico dall'Albania negli Stati Uniti. Ci pare giusto individuare il secondo tipo di viaggio di carattere metaforico, ossia quello di 
perdita di gender. Si tratta di un processo che dura nel tempo e assorda pian piano la vera identità della protagonista. Va però rilevato che il viaggio di perdita di gender non spegne totalmente la sua femminilità, soltanto la maschera, e quello di ritorno, la risveglia appieno per sempre. Ambedue i viaggi metaforici rilevano quanto siano reciprocamente dipendenti l'interiorità e il corpo.

\section{BIBLIOGRAFIA}

ABATE Carmine (2010), Il ballo tondo, Milano, Mondadori.

ABATE Carmine (2004), La festa del ritorno, Milano, Mondadori.

ADORNO Theodor (1999), Minima moralia. Refleksje z poharatanego życia, Kraków, Wydawnictwo Literackie.

ATKINS Dawn (1998), Looking queer: Image and Identity in Lesbian, Bisexual, Gay and transgendered Communities, Binghamton, NY, Haworth.

BAUMAN Zygmunt (1995), Ciało i przemoc w obliczu ponowoczesności, Toruń, Wydawnictwo Uniwersytetu Mikołaja Kopernika.

Bovo Romoeuf Martine (2008), L'epopea di Hora. La scrittura migrante di Carmine Abate, Firenze, Franco Cesati.

BUTLER Judith (2008), Uwikłani w płeć. Feminizm i polityka tożsamości, Warszawa, Wydawnictwo Krytyki Politycznej.

ChAncÉ Dominique (2005), Alterité, in : BENIAMINO M. e GAUVIN L. (ed.), Vocabulaire des études francophones, Limoges, Presse Universitaire de Limoges.

COMBERIATI Daniele (2013), Riscrivere la storia. Modalità di rappresentazione del colonialismo italiano in Albania, Incontri. Rivista europea di studi italiani 28, Igitur publishing, p. 25-33.

DONES Elvira (1997), Senza bagagli, Nardò, Besa.

DONES Elvira (2009), Vergine giurata, Milano, Feltrinelli.

FRATTA Carla (2001), Identità, in : ALBERTAZZI Silvia e VECCHI Roberto (a cura di), Abbecedario postcoloniale, Macerata, Quodlibet, p. 45-51.

FREUD Zygmunt (1992), Kultura jako źródło cierpień, Warszawa, Wydawnictwo KR.

FUCHS EPSTEIN Cynthia (2012), A theory of female subordination, in : LORBER Judith (ed.), Gender inequality. Feminist Theories and Politics, Oxford, Oxford University Press, p. 29-38.

FouCAult Michel (2000), Historia seksualności, Warszawa, Czytelnik.

GALIMBERTI Umberto (2003), Il corpo: antropologia, psicoanalisi, fenomenologia, Milano, Feltrinelli.

Grosz Elisabeth (1995), Space, Time and Perversion, Routledge, New York.

IBRAHIMI Anilda (2008), Rosso come una sposa, Einaudi, Torino.

KoŁAKOWSKI Leszek (1967), Kultura $i$ fetysze, Warszawa, Państwowe Wydawnictwo Naukowe.

KORNACKA Barbara (2013), Ucho, oko, ciato. O prozie „mtodych pisarzy” lat osiemdziesiatych i dziewięćdziesiątych we Włoszech, Poznań, Wydawnictwo UAM. 
KUPER Adam (2005), Kultura. Model antropologiczny, Kraków, Wydawnictwo Uniwersytetu Jagiellońskiego.

MAUCERI Maria Cristina (2009), Oltre il muro: dramma personale e nostalgia conflittuale in Dashuri e huaj di Elvira Dones, in : SiNOPOLI Franca (a cura di), La storia nella scrittura diasporica, Roma, Bulzoni, p. 85-107.

MEAD Margaret (1978), Kultura $i$ tożsamość. Studium dystansu międzypokoleniowego, Warszawa, Państwowe Wydawnictwo Naukowe.

MeAdOws Tay (2012), Rose is a rose, in : LORBER Judith (ed.), Gender inequality. Feminist Theories and Politics, Oxford, Oxford University Press, p. 289-303.

Moll Nora (2002), Immagini dell' "altro". Imagologia e studi interculturali, in : GNISCI Armando (a cura di), Letteratura comparata, Milano, Mondadori, p. $185-208$.

PeLLEGRINI Franca (2013), Traslazioni narrative: strategie di mediazione in Vergine giurata di Elvira Dones e Rosso come una sposa di Anilda Ibrahimi, in : BOND Emma e COMBERIATI Daniele (a cura di), Il confine liquido. Rapporti letterari e interculturali fra Italia e Albania, Nardò, Besa, p. 149166.

RICEUUR Paul (1990), Soi-même comme un autre, Paris, Seuil.

SERKOWSKA Hanna (2005), Reprezentacje inności w prozie włoskiej dwudziestego wieku, in : OSTROWSKA Elżbieta e DURYS Elżbieta (ed.), Wizerunki kobiet i mężczyzn w kulturze, Kraków, Rabid.

VORPSI Ornela (2005), Il paese dove non si muore mai, Torino, Einaudi.

VORPSI Ornela (2012), Fuorimondo, Torino, Einaudi. 